\title{
The Rotary Cell Culture System increases NTRK3 Expression and Promotes Neuronal Differentiation and Migratory Ability of Neural Stem Cells Cultured on Collagen Sponge
}

Yi Cui

National Research Institute for Family Planning

Yanyun Yin

Institute of Genetics and Developmental Biology CAS: Institute of Genetics and Developmental Biology Chinese Academy of Sciences

\section{Yannan Zhao}

Institute of Genetics and Developmental Biology CAS: Institute of Genetics and Developmental Biology Chinese Academy of Sciences

\section{Jin Han}

Institute of Genetics and Developmental Biology CAS: Institute of Genetics and Developmental Biology Chinese Academy of Sciences

\section{Bai Xu}

Institute of Genetics and Developmental Biology CAS: Institute of Genetics and Developmental Biology Chinese Academy of Sciences

\section{Yunlong Zou}

China-Japan Union Hospital of Jilin University

\section{Bing Chen}

CAS Institute of Genetics and Developmental Biology: Institute of Genetics and Developmental Biology Chinese Academy of Sciences

\section{Zhifeng Xiao}

CAS Institute of Genetics and Developmental Biology: Institute of Genetics and Developmental Biology Chinese Academy of Sciences

\section{Hongwei Song}

EHBIO gene technology

\section{Ya Shi}

CAS Institute of Genetics and Developmental Biology: Institute of Genetics and Developmental Biology Chinese Academy of Sciences

\section{Weiwei Xue}

CAS Institute of Genetics and Developmental Biology: Institute of Genetics and Developmental Biology Chinese Academy of Sciences 


\section{Xu Ma}

National Research Institute for Family Planning

Jianwu Dai ( $\nabla$ xda_dai@genetics.ac.cn )

Institute of Genetics and Developmental Biology Chinese Academy of Sciences

\section{Research}

Keywords: RCCS, NSCs, NTRK3, let-7i-5p, collagen sponge scaffold

Posted Date: December 1st, 2020

DOl: https://doi.org/10.21203/rs.3.rs-115776/v1

License: (c) (1) This work is licensed under a Creative Commons Attribution 4.0 International License. Read Full License 


\section{Abstract}

Background Recently, neural stem cell (NSC) therapy has shown promise for the treatment of many neurological diseases. Enhancing the quality of implanted cells and improving therapeutic efficacy are currently research hotspots. It has been reported that collagen sponge material provided sufficient room for cell growth in all directions, promoted the absorption of nutrients and removal of wastes. And also, the Rotary Cell Culture System (RCCS), which mimics the microgravity environment, can be used to culture cells for tissue engineering.

Materials and methods We performed the mRNA and miRNA sequencing to elucidate the regulatory mechanism of NSCs cultured on the collagen sponge in RCCS system. The luciferase assay and Western blot revealed a direct regulatory role between let-7i-5p and NTRK3. And then, the neural differentiation maker Tuj1 and Map2 were detected by immunofluorescence staining. In the meantime, the migratory ability of NSCs was detected both in vitro and in spinal cord injury animals.

Results In this study, we demonstrated that the expression of neurotrophic receptor tyrosine kinase 3 (NTRK3; also called TrkC) was elevated in NSCs cultured on collagen sponge in the RCCS system. Furthermore, increased NTRK3 expression was regulated by the downregulation of let-7i-5p. Compared to traditionally cultured NSCs, the NSCs cultured on collagen sponge in the RCCS system exhibited better neuronal differentiation and migratory ability, especially in the presence of NT-3.

Conclusions As the biological properties and quality of transplanted cells are critical for therapeutic success, the RCCS system combined with collagen sponge culture system show promise for applications in clinical practice in the future.

\section{Full Text}

This preprint is available for download as a PDF.

\section{Figures}


(A)

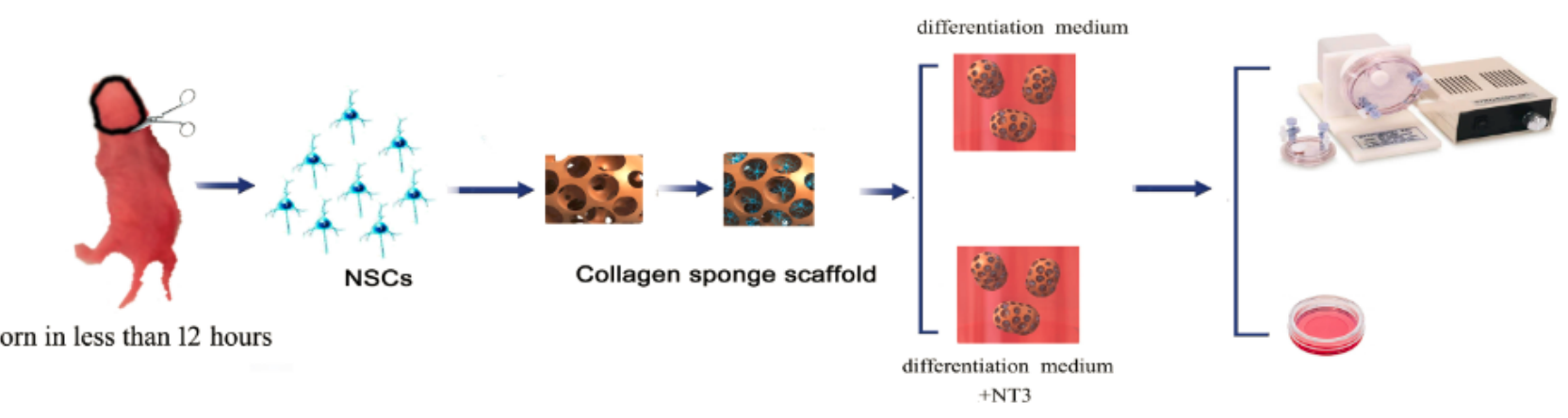

(B)
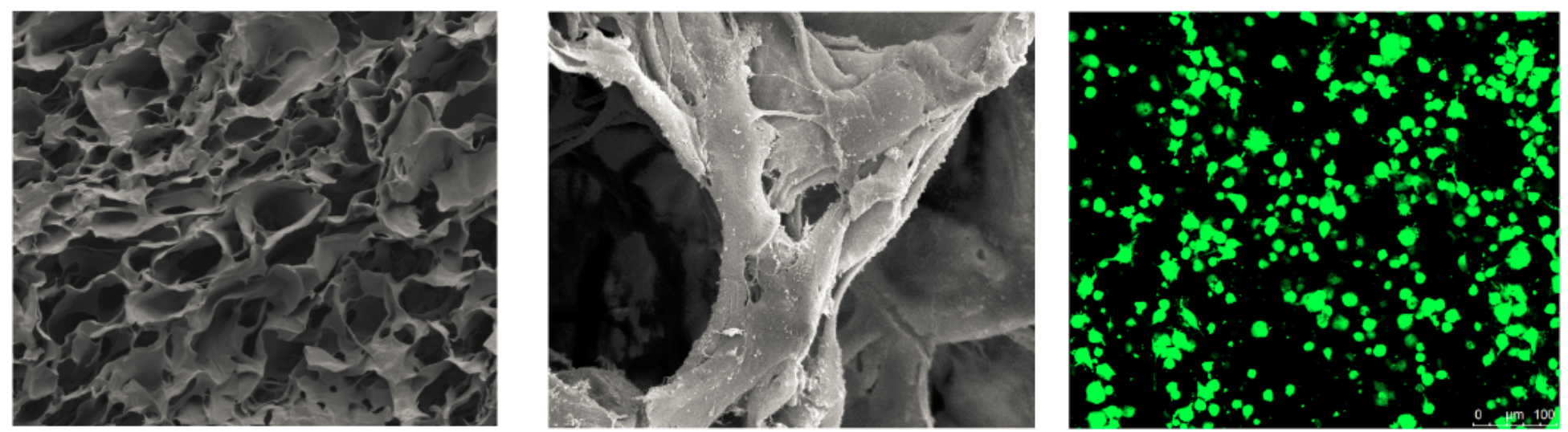

Figure 1

(A) A flowchart of the experiment. (B) Scanning electron microscopy revealed the morphology of the collagen sponge scaffold (left) and 3D cultured NSCs (middle); fluorescein diacetate staining of the 3D cultured NSCs (right). 
(A)
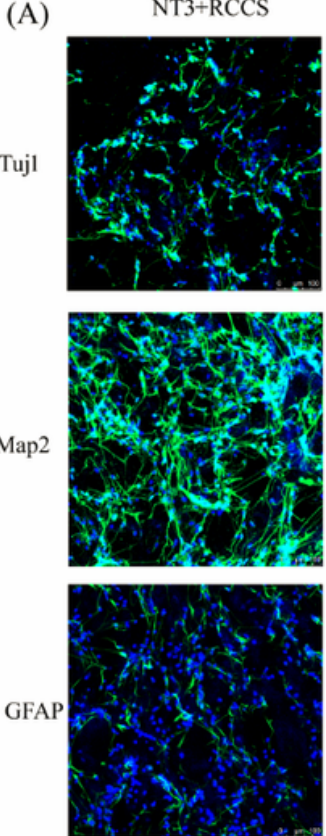

RCCS
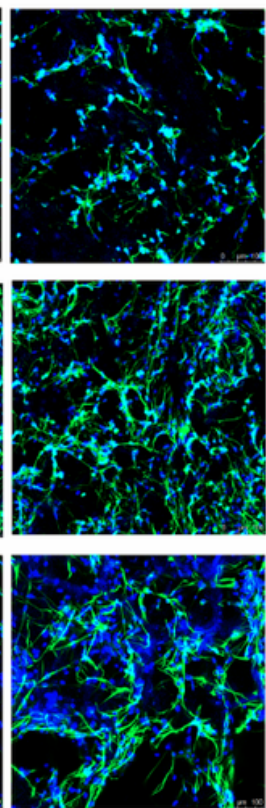

static
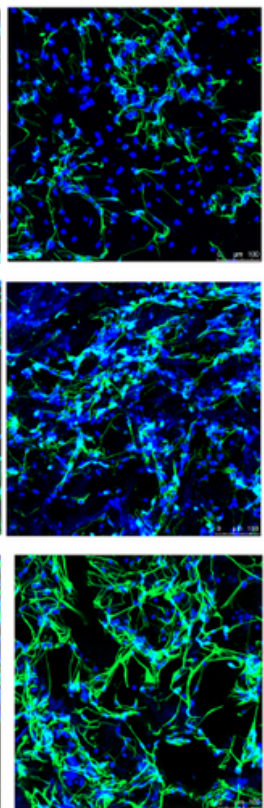

$\mathrm{NT} 3+$ static
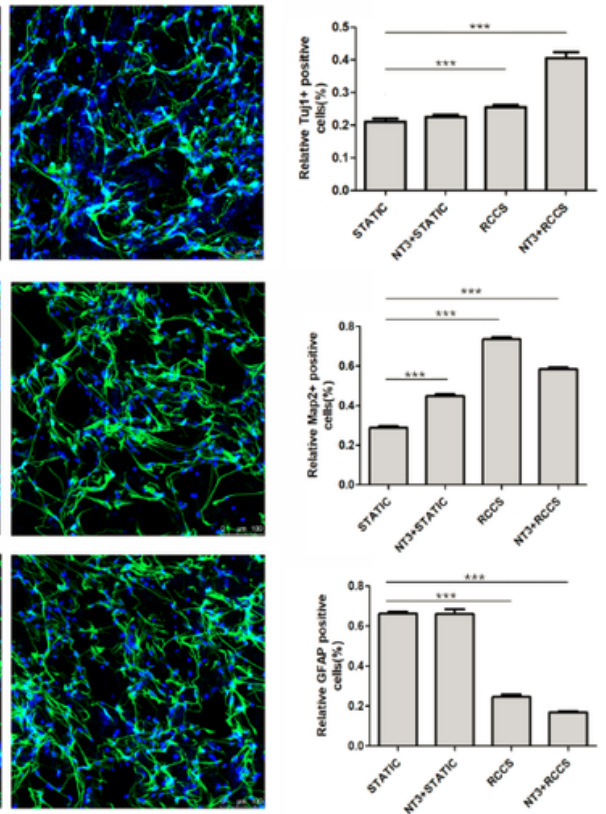

(B)
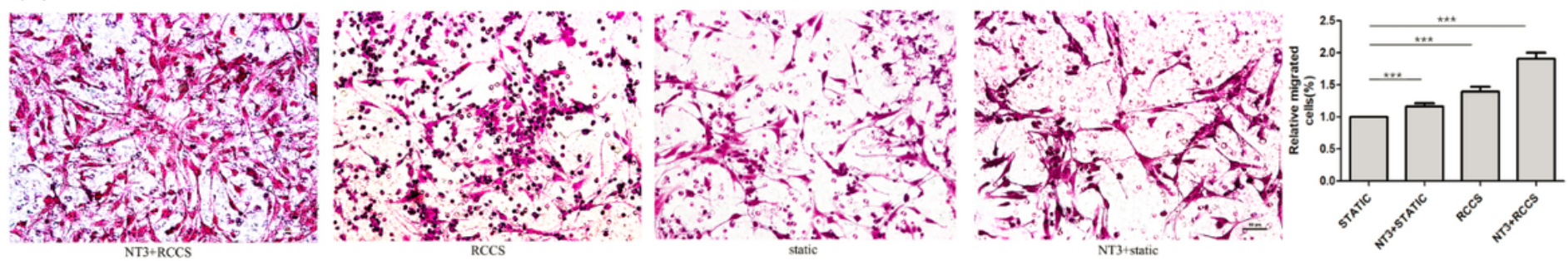

Figure 2

The differentiation and migratory ability of 3D cultured NSCs in all groups detected by immunofluorescence staining (A) and trans-well migration array (B).
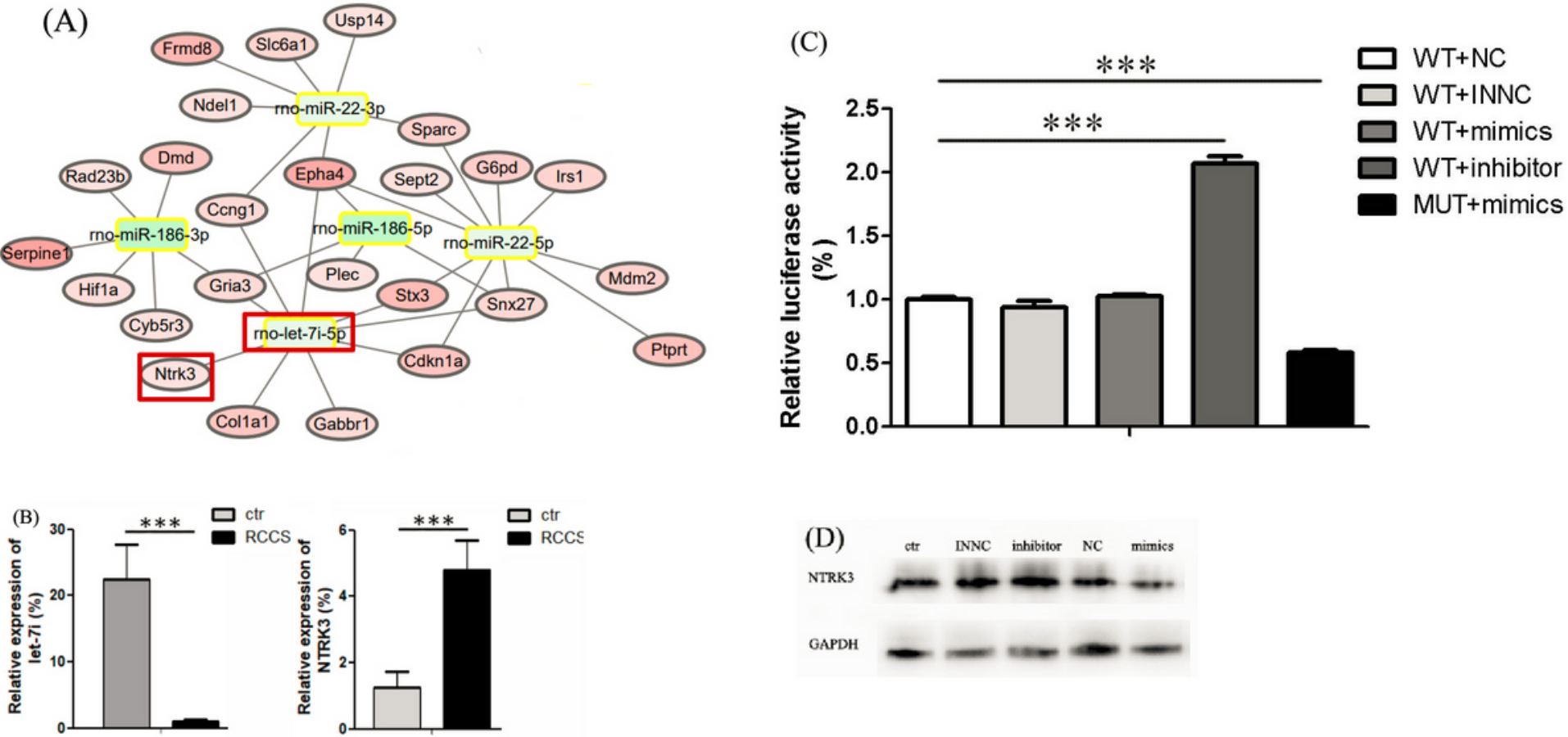


\section{Figure 3}

The interaction between let-7i-5p and NTRK3. (A) Bioinformatics analysis predicted a regulatory role of let-7i-5p on NTRK3. (B) Real-time PCR indicates let-7i-5p and NTRK3 expression in the RCCS and control groups. (C) The luciferase reporter array indicated an interaction between 3'-UTR of NTRK3 and the seed sequence of let-7i-5p. (D) Western blot indicated that NTRK3 protein levels are affected by let-7i-5p mimics and inhibitors.

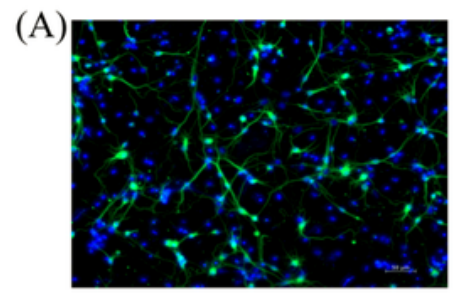

CTR

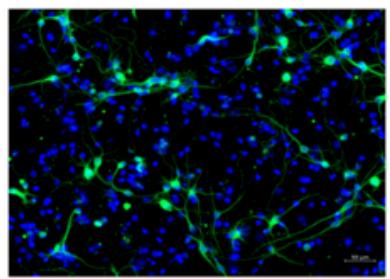

let-7i-inhibitor + TrkC siRNA

(B)

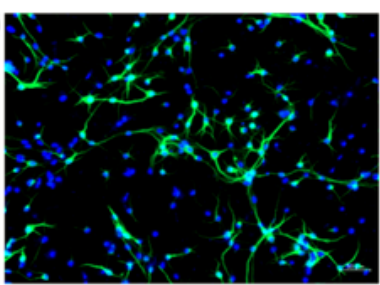

CTR

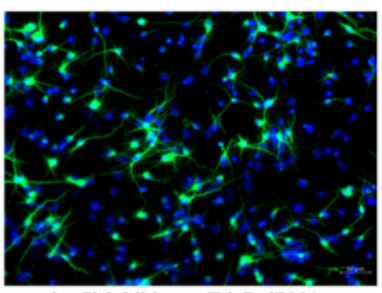

let-7i-inhibitor + TrkC siRNA

(C)

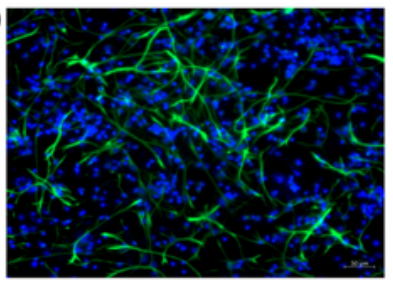

CTR

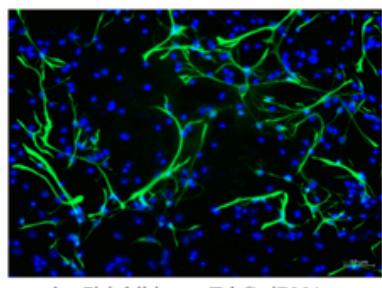

let-7i-inhibitor + TrkC siRNA

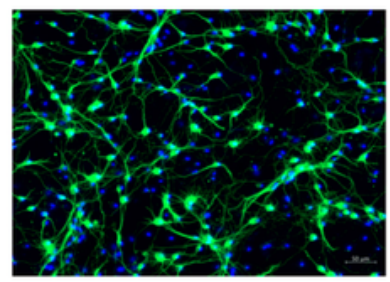

let-7i-inhibitor

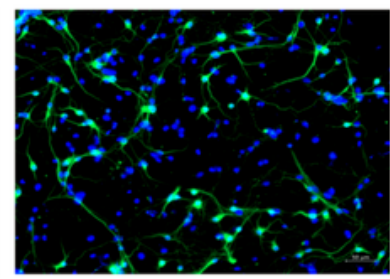

let-7i-mimics

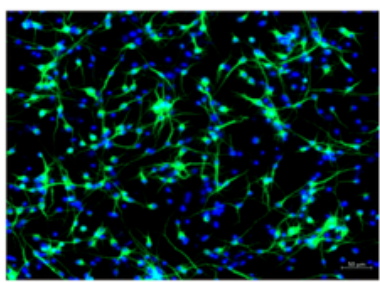

let-7i-inhibitor

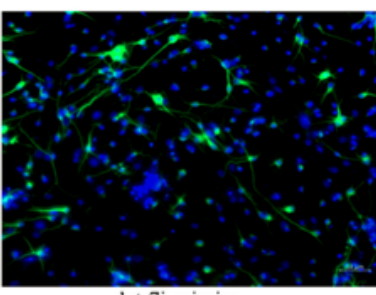

let-7i-mimics

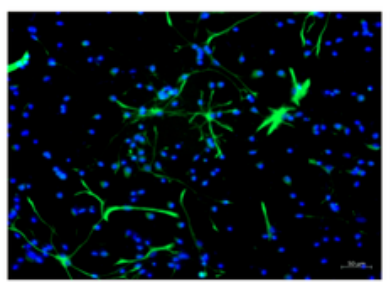

let-7i-inhibitor

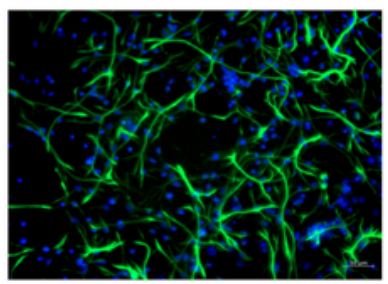

let-7i-mimics

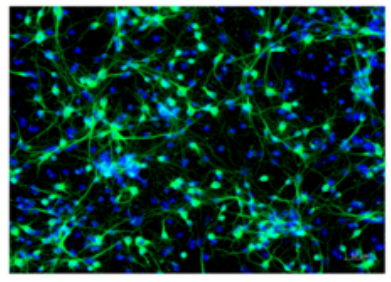

TrkC overexpression

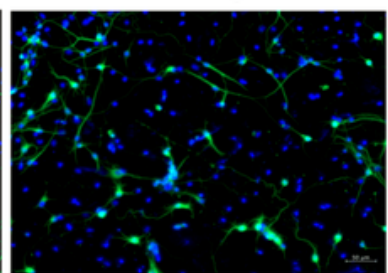

TrkC siRNA

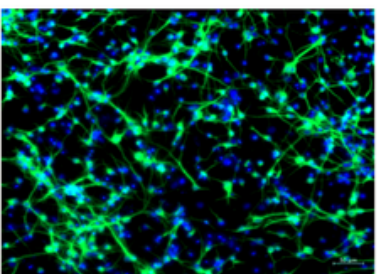

TrkC overexpression

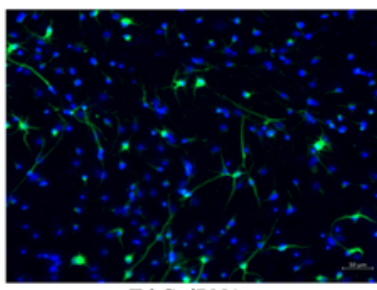

TrkC siRNA

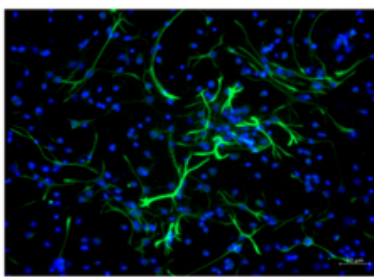

TrkC overexpression

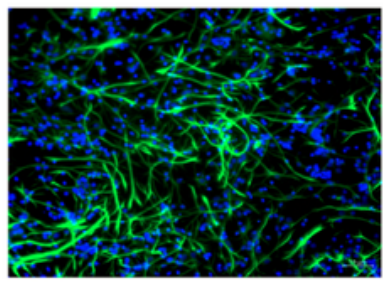

TrkC siRNA 


\section{Figure 4}

Immunofluorescence staining indicated an effect of let-7i-5p and NTRK3 on NSC differentiation when the differentiation medium contained NT3; (A) Tuj1(Neuronal Class III $\beta$-Tubulin), (B) Map2 (microtubule associated protein 2), (C) GFAP (glial fibrillary acidic protein).

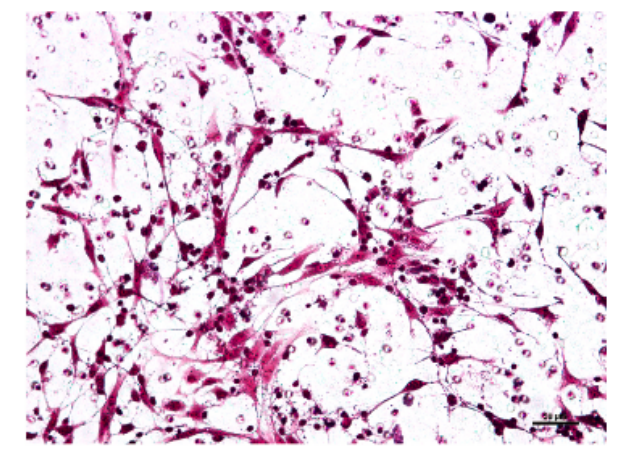

CTR

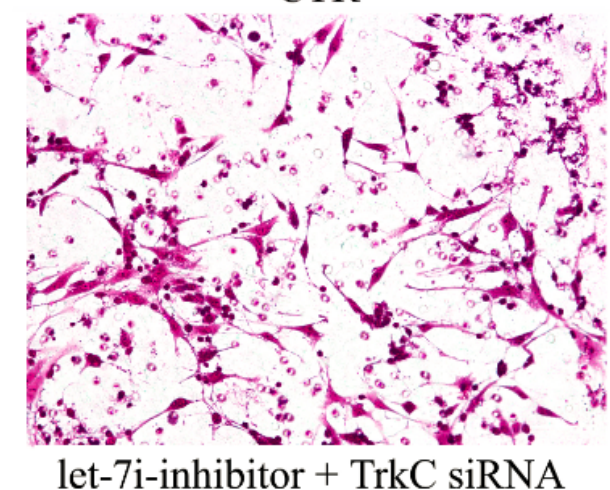

let-7i-inhibitor + TrkC siRNA

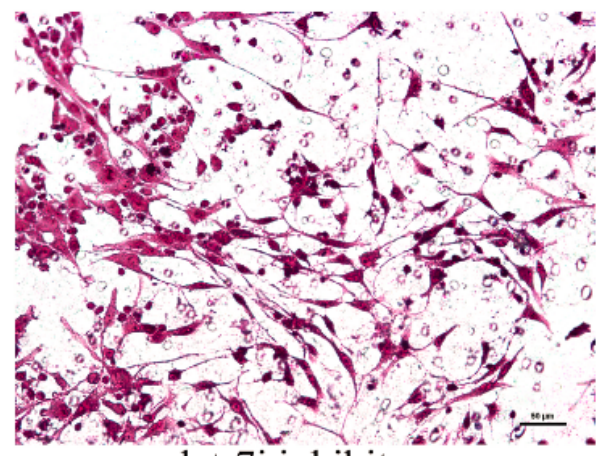

let-7i inhibitor

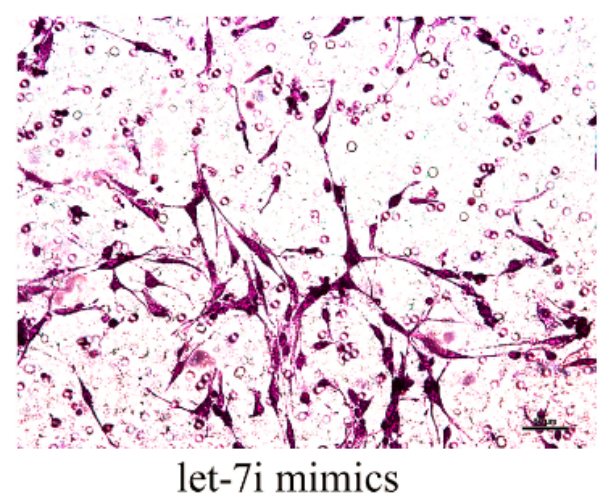

let-7i mimics

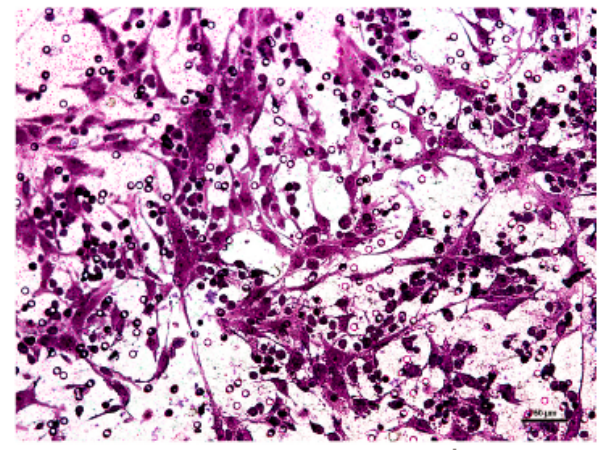

NTRK3 overexpression

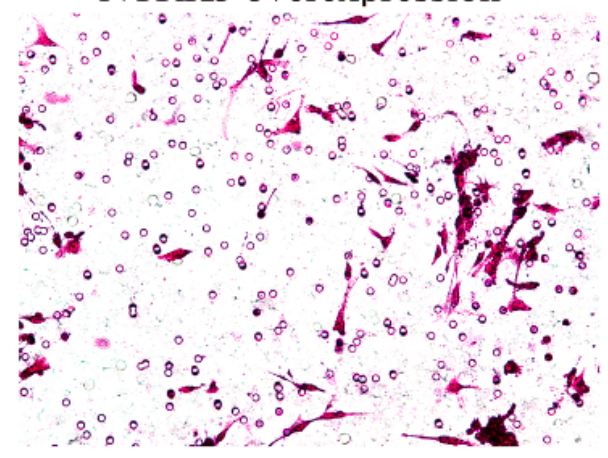

NTRK3 siRNA

Figure 5

The transwell migration assay indicated an effect of let-7i-5p and NTRK3 on NSC migration in the presence of NT3. 
(A)
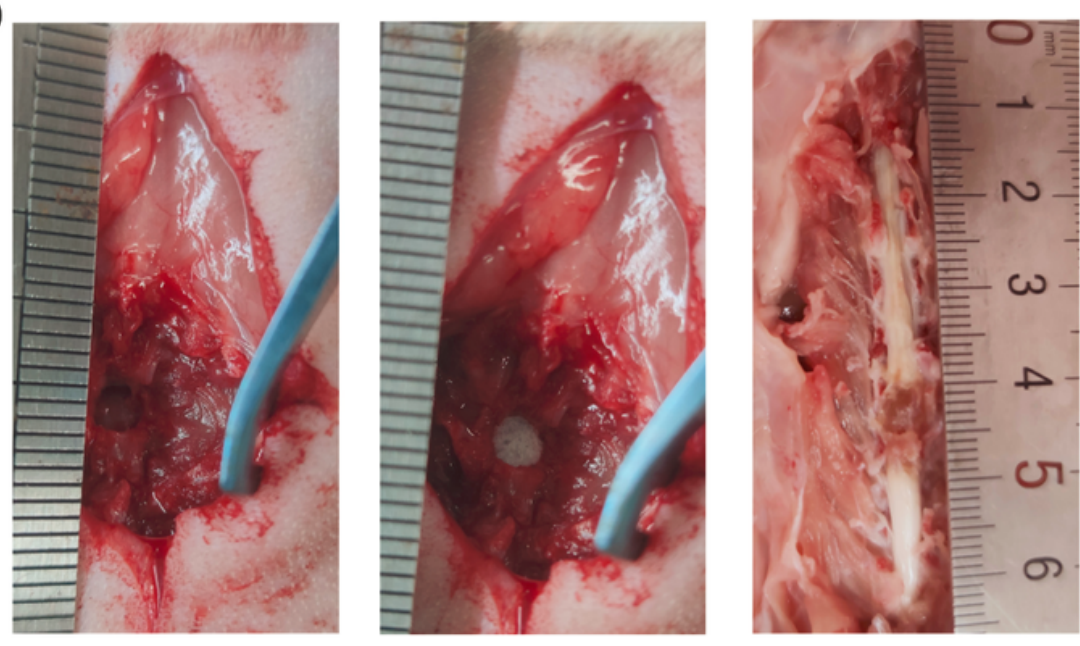

(B)

static

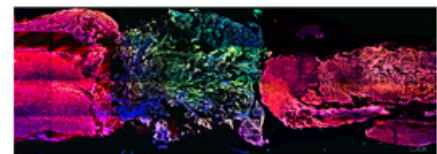

RCCS

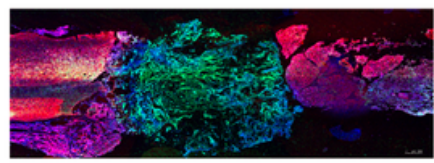

RCCS+NT3

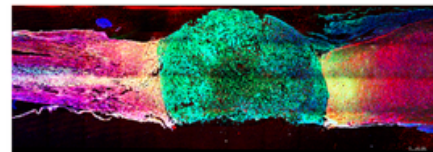

(C)

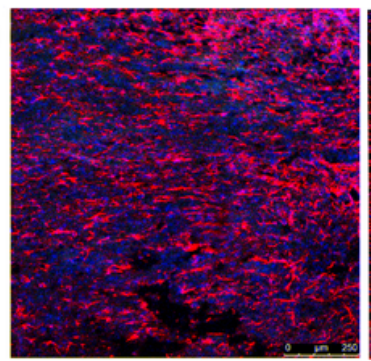

static

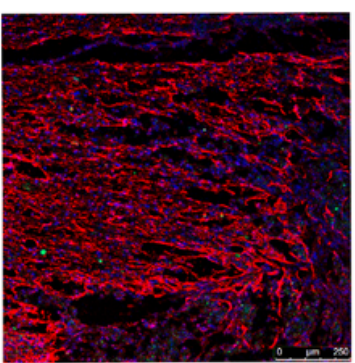

static + NT3

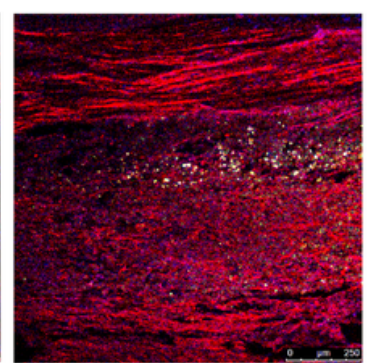

RCCS

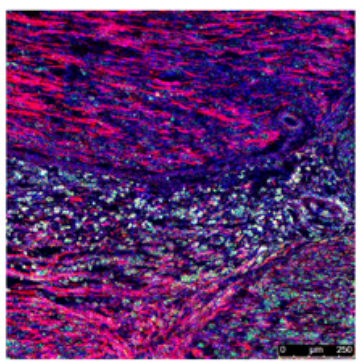

$\mathrm{RCCS}+\mathrm{NT} 3$

\section{Figure 6}

The transplantation of NSCs in rats with spinal cord injury. (A) Spinal cord injuries (with lengths of $3 \mathrm{~mm}$ ) were inflicted in rats (left). Functional collagen sponge scaffolds with or without cells were precisely placed into the injury site (middle). Samples of the spinal cord tissue following perfused $4 \%$ paraformaldehyde fixation (right). (B) GFAP immunostaining in all groups. The green signal represents the GFP-labelled transplanted NSCs. (C) Enlarged images of the migrated GFP+ cells in the RCCS and RCCS+NT-3 groups. 